\title{
2'-Hydroxyflavanone inhibits prostate tumor growth through inactivation of AKT/STAT3 signaling and induction of cell apoptosis
}

\author{
KAIJIE WU ${ }^{1 *}$, ZHONGYUN NING $^{1,2^{*}}$, JIANCHENG ZHOU $^{1,2}$, BIN WANG $^{1,2}$, \\ JINHAI FAN ${ }^{1}$, JIANNING ZHU ${ }^{1,2}$, YANG GAO ${ }^{1,2}$, XINYANG WANG ${ }^{1,2}$, \\ JER-TSONG HSIEH ${ }^{3}$ and DALIN HE ${ }^{1,2}$
}

\author{
${ }^{1}$ Department of Urology, First Affiliated Hospital of the Medical School, Xi'an Jiaotong University; \\ ${ }^{2}$ Oncology Research Laboratory, Key Laboratory of Environment and Genes Related to Diseases, \\ Ministry of Education of the People's Republic of China, Xi'an, Shaanxi 710061, P.R. China; \\ ${ }^{3}$ Department of Urology, University of Texas Southwestern Medical Center, Dallas, TX 75390, USA
}

Received January 28, 2014; Accepted April 11, 2014

DOI: $10.3892 /$ or.2014.3218

\begin{abstract}
Although there have been advances in therapeutic regimes for metastatic castration-resistant prostate cancer (CRPC), these recent developments have not led to improved cure rates. Thus, more novel agents to prolong patient survival are desired. 2'-Hydroxyflavanone (2HF), a nontoxic natural flavonoid, has been shown to exhibit pleiotropic anticancer effects in many cancer types, including prostate cancer (PCa). However, the therapeutic effects of $2 \mathrm{HF}$ on tumor growth and its potential mechanisms in CRPC have not been completely elucidated. In the present study, utilizing three different metastatic and androgen-independent PCa cell models (PC-3, DU145 and C4-2), we found that $2 \mathrm{HF}$ treatment not only resulted in inhibition of cell proliferation and colony formation in vitro, but also delayed subcutaneous tumor growth in vivo. Mechanistically, besides its known inhibitory effects on aldo-keto reductase activity and de novo androgen synthesis, $2 \mathrm{HF}$ also markedly suppressed AKT phosphorylation, signal transducer and activator of transcription-3 (STAT3) phosphorylation and transactivation subsequently regulating the expression of members of the BCL-2 family (i.e., Mcl-1, Bcl-2 and Bax) and modulating caspase-mediated cell apoptosis. Overall, this study revealed a novel mechanism for $2 \mathrm{HF}$ targeting metastatic CRPC, in which inactivation of AKT/STAT3 signaling led to cell apoptosis and growth inhibition.
\end{abstract}

Correspondence to: Professor Dalin He, Department of Urology, First Affiliated Hospital of the Medical School, Xi'an Jiaotong University, 277 Yanta West Road, Xi'an, Shaanxi 710061, P.R. China E-mail: dalinhexjtu@126.com

*Contributed equally

Key words: 2'-hydroxyflavanone, prostate cancer, cell apoptosis, AKT, STAT3

\section{Introduction}

Prostate cancer (PCa) is the most common type of cancer and the second leading cause of cancer-related mortality in men in North America (1). Since many patients have already developed metastatic lesions at initial clinical presentation, hormone therapy has become a standard treatment with confirmed beneficial effects for hormone-sensitive disease. However, castration-resistant prostate cancer (CRPC) invariably recurs within 1 to 2 years, and few treatment options are available once this aggressive prostate cancer has the capacity to resist chemotherapy or radiotherapy (2-4). Therefore, novel adjuvant agents with complete efficacy and low toxicity are desired to treat metastatic CRPC and substantially prolong patient survival.

Flavonoids, consisting of flavones, flavanones, isoflavones and flavanols, are important for human nutrition and plant biology as plant secondary metabolites. Several isolated naturally existing flavanones have been determined for their anti-inflammatory, anti-oxidative and anti-bacterial activities (5).In addition, structure-activity studies have demonstrated that flavanones with more hydroxyl groups exhibit increased bioactivities (6). Moreover, hydroxyflavanones have also been well characterized to have various antitumor properties via distinct mechanisms of action $(7,8)$. For example, flavanones and 2'-hydroxyflavanone (2HF, chemical structure as shown in Fig. 1A) inhibit the metastasis of lung cancer cells via downregulation of proteinase activities and the MAPK pathway (9). $2 \mathrm{HF}$ was also found to inhibit proliferation, tumor vascularization and to promote normal differentiation in VHL-mutant renal cell carcinoma (10). Moreover, $2 \mathrm{HF}$ induced apoptosis through Egr-1 involving expression of Bax, p21 and NAG-1 in colon cancer cells (11). However, few studies have shown the therapeutic effects and potential mechanisms of $2 \mathrm{HF}$ in regards to the growth of prostate cancer, particularly CRPC.

In the present study, utilizing three different metastatic and androgen-independent PCa cell models (PC-3, DU145 
and C4-2), we showed that $2 \mathrm{HF}$ treatment significantly suppressed cell proliferation and clonogenicity in a dose- and time-dependent manner. It also markedly inhibited subcutaneous tumor growth in vivo. Furthermore, $2 \mathrm{HF}$ enhanced cell apoptosis in a dose-dependent manner, which was confirmed by Annexin V/propidium iodide (PI) staining and cleavage of poly(ADP-ribose) polymerase (PARP) and caspase-3. Mechanistically, 2HF induced AKT dephosphorylation, inhibited the phosphorylation and transactivation of signal transducer and activator of transcription-3 (STAT3) and subsequently downregulated the expression of its downstream target genes (i.e., Mcl-1, Bcl-2 and Bax), which are probably involved in the regulation of apoptotic and anti-apoptotic pathways.

\section{Materials and methods}

Cell culture and $2 H F$ treatment. Androgen receptor (AR)-negative PCa cell lines PC-3 (bone metastatic) and DU145 (brain metastatic) were maintained in Dulbecco's modified Eagle's medium (DMEM; Gibco, San Diego, CA, USA) supplemented with $10 \%$ fetal bovine serum (FBS) at $37^{\circ} \mathrm{C}$ with $5 \% \mathrm{CO}_{2}$ in a humidified incubator. Androgensensitive but independent AR-positive PCa cell line C4-2 (bone metastatic) was maintained in T-medium supplemented with 5\% FBS. 2HF was obtained from Sigma (St. Louis, MO, USA) and dissolved in dimethyl sulfoxide (DMSO). For $2 \mathrm{HF}$ treatment, a stock solution (0.02 M in DMSO) was added into the culture medium with $1 \%$ FBS to achieve the appropriate concentration $(10,20$ or $40 \mu \mathrm{mol} / \mathrm{l})$ and then incubated with the cells, whereas DMSO solution without $2 \mathrm{HF}$ was used as the control.

Cell viability assay. To evaluate the sensitivities of different prostate cancer cells to $2 \mathrm{HF}$ treatment, 3-(4,5-dimethylthiazol-2-yl)-2,5-diphenyltetrazolium bromide (MTT; Sigma) proliferation assays were performed to determine cell viability. Different PCa cells were plated at a density of $2 \times 10^{3}$ cells/well in 96-well plates for $24 \mathrm{~h}$ and then treated with different concentrations of $2 \mathrm{HF}(0,10,20$ and $40 \mu \mathrm{M})$ for the indicated time. After the exposure period, $20 \mu \mathrm{MTT}$ was added to each well for another $5 \mathrm{~h}$ incubation at $37^{\circ} \mathrm{C}$ in $5 \% \mathrm{CO}_{2}$. Thereafter, the medium containing MTT was removed, and $150 \mu 1$ DMSO was added to solubilize the formazan crystals. The absorbance (OD) was then measured at a wavelength of $590 \mathrm{~nm}$ by a microplate autoreader (Bio-Tek Instruments, Vinooski, VT, USA). Independent experiments were repeated in triplicate.

Colony formation assays. The colony formation assay was used to determine the clonogenicity capabilities in vitro as described previously (12). The indicated cells were treated with different concentrations of $2 \mathrm{HF}(0,10,20$ and $40 \mu \mathrm{M})$ for $36 \mathrm{~h}$. Then an equal number of cells were seeded in 6-well plates to form colonies for at least two weeks and fresh medium was changed every 3-4 days. The plates were then washed with ice-cold PBS, fixed with $4 \%$ paraformaldehyde, stained in crystal violet solution for $15 \mathrm{~min}$ at room temperature and washed with distilled water to remove excess dye. The number of colonies was counted for each sample.
Flow cytometric analysis for cell apoptosis. PC-3, DU145 or C4-2 cells $\left(1-3 \times 10^{6}\right)$ were plated on a $6-\mathrm{cm}$ dish for $24 \mathrm{~h}$, and treated with different concentrations of $2 \mathrm{HF}(0,10,20$ and $40 \mu \mathrm{M}$ ) for another $24 \mathrm{~h}$. After that, cells were harvested, washed with PBS and then subjected to Annexin V and PI staining using an Annexin V-FITC apoptosis detection kit (Invitrogen, Eugene, OR, USA) according to the manufacturer's instructions and immediately analyzed by flow cytometry (FACSCalibur, BD Biosciences, NJ, USA).

Western blot analyses. The indicated cells were treated with $2 \mathrm{HF}$ for $36 \mathrm{~h}$ and then total cellular protein lysates were prepared with RIPA buffer [50 mM Tris (pH 8.0), $150 \mathrm{mM}$ $\mathrm{NaCl}, 0.1 \%$ SDS, $1 \%$ NP-40 and $0.5 \%$ sodium deoxycholate] containing proteinase inhibitors, $1 \%$ Cocktail and $1 \mathrm{mmol} / \mathrm{l}$ PMSF (Sigma, St. Louis, MO, USA). A total of 20-40 $\mu \mathrm{g}$ of protein was separated by $10-12 \%$ SDS-PAGE and transferred to nitrocellulose membranes. Following blocking of the nonspecific binding sites on the membranes with 5\% skimmed milk in Tris-buffered saline with $0.1 \%$ Tween-20 (pH 7.6, TBST) for $1 \mathrm{~h}$ at room temperature, the membranes were then incubated with the desired primary antibody overnight at $4^{\circ} \mathrm{C}$. Antibodies for western blotting were rabbit anti-PARP (CST9532; Cell Signaling Technology, Danvers, MA, USA) at a 1:500 dilution, rabbit anti-phosphorylated-AKT (Ser473, CST-4060, 1:1,000), rabbit anti-AKT (CST-9272, 1:1,000), mouse antiphosphorylated-STAT3 (Tyr705, CST-4113, 1:1,000), rabbit anti-STAT3 (CST-8232, 1:1,000), rabbit anti-Mcl-1 (CST-5453, 1:500), rabbit anti-Bcl-2 (SC-492; Santa Cruz Biotechnology, Santa Cruz, CA, USA) at a 1:500 dilution, rabbit anti-Bax (SC-493, 1:200) and rabbit anti-caspase-3 (CST-9665, 1:1,000). After being washed with TBST, membranes were incubated with secondary antibodies coupled to horseradish peroxidase at room temperature for $1 \mathrm{~h}$ and visualized with an ECL chemiluminescent detection system (Pierce, Rockford, IL, USA). Loading differences were normalized using monoclonal glyceraldehyde-3-phosphate dehydrogenase (GAPDH) antibody.

Dual-luciferase reporter assay. For the reporter gene assay, cells seeded in 24-well plates were transfected with $200 \mathrm{ng}$ STAT3-responsive luciferase reporter plasmid pLucTKS3 or the control plasmid pLucTK (13) and $1 \mathrm{ng}$ of the pRL-SV40 Renilla luciferase construct (as an internal control) for $24 \mathrm{~h}$ and then subjected to 2HF, PI3K inhibitor LY 294002 or STAT3 inhibitor Stattic treatment. Cell extracts were prepared $36 \mathrm{~h}$ after treatment and the luciferase activity was measured using the Dual-Luciferase reporter assay system (Promega) as described previously (14). Relative luciferase activity is represented as mean \pm SEM from each sample after normalizing with the control.

In vivo subcutaneous xenograft model. Sixteen male BALB/ c-nu mice at the age of 3-4 weeks and weighing 15-20 g were purchased from the Shanghai Laboratory Animal Center (SLAC, Shanghai, China) and were acclimated for one week before beginning the experiment. All animal experiments were carried out in accordance with a protocol approved by the Institutional Animal Care and Use Committee. Single cells $\left(5 \times 10^{6}\right)$ were subcutaneously inoculated into both flanks 
A<smiles>O=C1CC(c2ccccc2Cl)Oc2ccccc21</smiles>

2‘-Hydroxyflavanone (2HF)

C
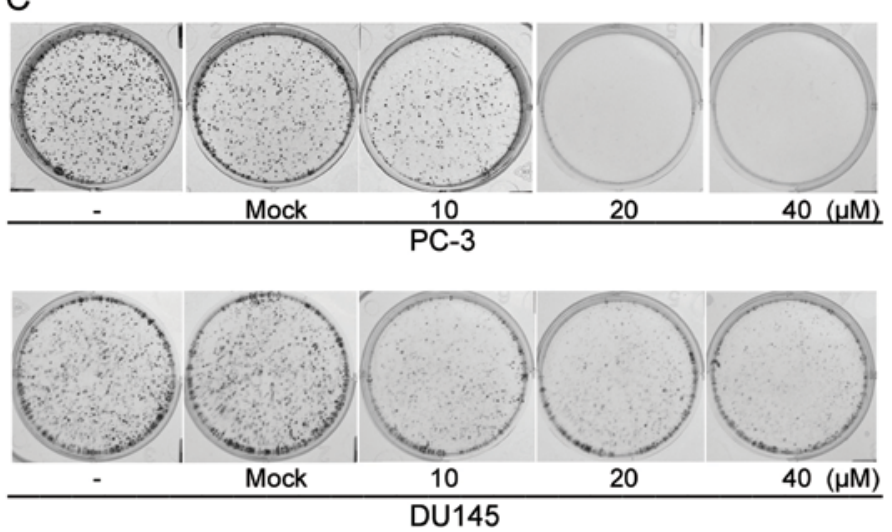

D
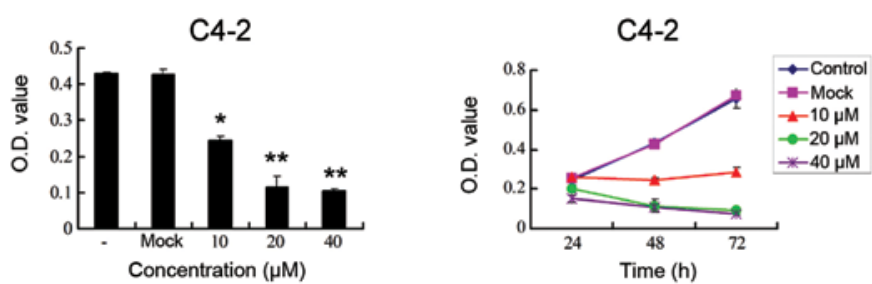

B

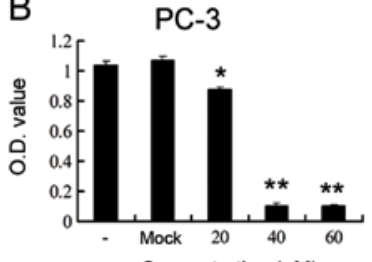

Concentration $(\mu \mathrm{M})$
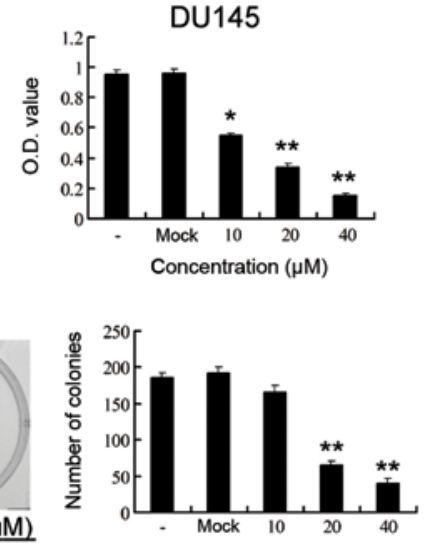

Concentration $(\mu \mathrm{M})$

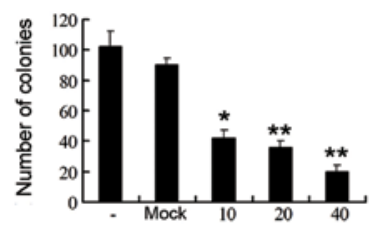

Concentration $(\mu \mathrm{M})$

Figure 1. 2HF inhibits cell proliferation and clonogenicity of metastatic CRPC cells in vitro. (A) Chemical structure of 2HF. (B) PC-3 and DU145 cells were treated with different doses of $2 \mathrm{HF}(0,20$ and $40 \mu \mathrm{mol} / \mathrm{l})$ for $48 \mathrm{~h}$ and processed for MTT assay to measure the changes in cell proliferation. Compared with the control, ${ }^{*} \mathrm{P}<0.05,{ }^{* *} \mathrm{P}<0.01$. (C) PC-3 and DU145 cells were treated with different doses of $2 \mathrm{HF}(0,20$ and $40 \mu$ mol $/ 1)$ for $48 \mathrm{~h}$ and processed for colony formation assay to measure the changes in cell clonogenicity. Left panel, representative images of colony formation after $2 \mathrm{HF}$ treatment are shown. Right panel, quantitative analysis is shown. Compared with the control, ${ }^{*} \mathrm{P}<0.05,{ }^{* *} \mathrm{P}<0.01$. (D) Left panel, C4-2 cells were treated with different doses of $2 \mathrm{HF}(0,20$ and $40 \mu \mathrm{mol} / \mathrm{l}$ ) for $48 \mathrm{~h}$ and processed for MTT assay to measure the changes in cell proliferation. Compared with the control, ${ }^{*} \mathrm{P}<0.05$, ${ }^{* *} \mathrm{P}<0.01$. Right panel, C4-2 cells were treated with different doses of $2 \mathrm{HF}(0,20$ and $40 \mu \mathrm{mol} / \mathrm{l})$ for the indicated times before MTT assay and the growth curve was analyzed.

of the mice. Once the tumors were established $\left(100-150 \mathrm{~mm}^{3}\right)$, the mice were randomly divided into two groups. The mice (eight in each group) received $2 \mathrm{HF}$ (100 $\mathrm{mg} / \mathrm{kg}$ body weight, daily) in $100 \mu \mathrm{l}$ corn oil by oral gavage once per day. Control groups were treated with corn oil only. Body weight and tumor volume were measured two times per week. Calipers were used to measure the two dimensions of the tumors. Thirty days after treatment, mice were sacrificed by cervical dislocation, and tumors were removed and weighed.

Immunohistochemical (IHC) staining. Xenografts were harvested for staining and IHC was carried out with Dako Autostainer Plus system (Dako, Carpinteria, CA, USA) as previously described (14). Briefly, sections were deparaffinized, rehydrated and subjected to antigen retrieval in citrate buffer $(10 \mathrm{mM}, \mathrm{pH} 6.0)$ for $5 \mathrm{~min}$ and then endogenous peroxidase and alkaline phosphatase activities were blocked with Dual Block for $10 \mathrm{~min}$. The slides were then incubated overnight at $4^{\circ} \mathrm{C}$ with p-AKT (Ser473, CST-4060), p-STAT3 (Tyr705, CST-4113), Bcl-2 (SC-492) and cleaved caspase-3 (CST-9661) antibodies (1:75 dilution). After washing, this was followed by incubation with EnVision secondary antibody for $30 \mathrm{~min}$ at room temperature. Signals were detected by adding substrate hydrogen peroxide using diaminobenzidine (DAB) as a chromogen followed by hematoxylin counterstaining.

Statistical analysis. All assays were repeated in triplicate in three independent experiments and quantitative data are presented as means \pm SEM. The differences between two groups were compared by the 2-tailed Student's t-test. In all cases, a P-value of $<0.05$ was considered to indicate a statistically significant difference. All statistical analyses were performed using SPSS 18.0 (SPSS Inc, Chicago, IL, USA).

\section{Results}

$2 H F$ inhibits cell proliferation and clonogenicity of prostate cancer cells in vitro. The progression to androgen independence is a multi-factorial process by which cells acquire the ability to both survive in the absence of androgens and proliferate using non-androgenic stimuli for mitogenesis. Therefore, targeting tumor cell growth may still play an important role in CRPC therapy. As shown in Fig. 1B, we initially showed that 2HF inhibited in vitro cell proliferation in a dose-dependent manner in PC-3 cells, which were derived from bone metastatic lesion and are androgen-independent. Furthermore, we 
A

PC-3
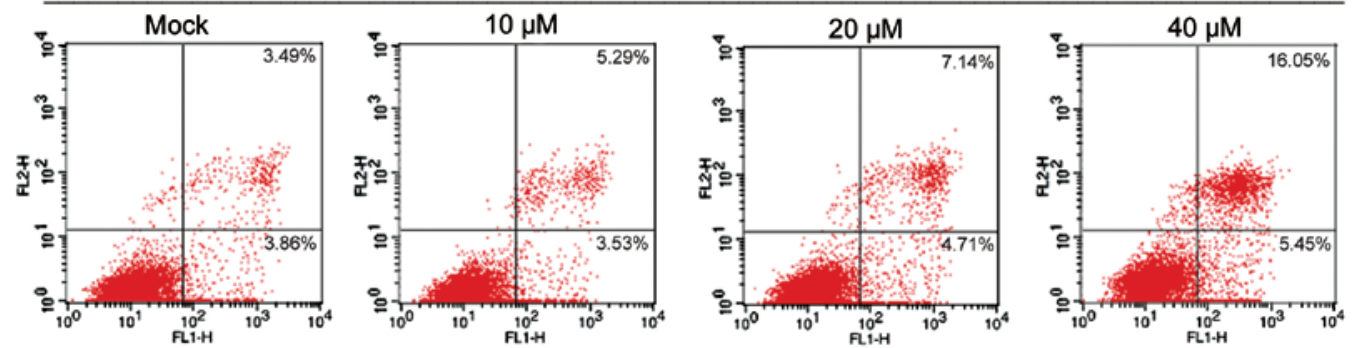

B

DU145
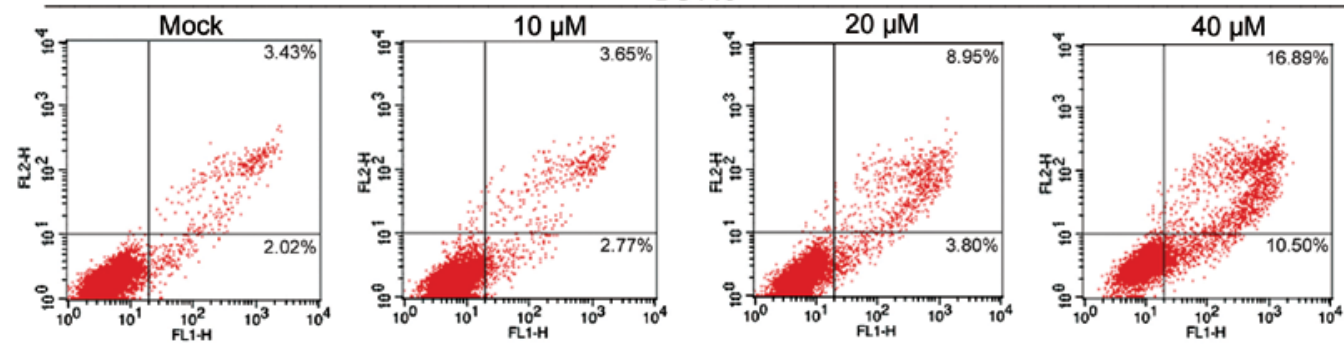

C

C4-2
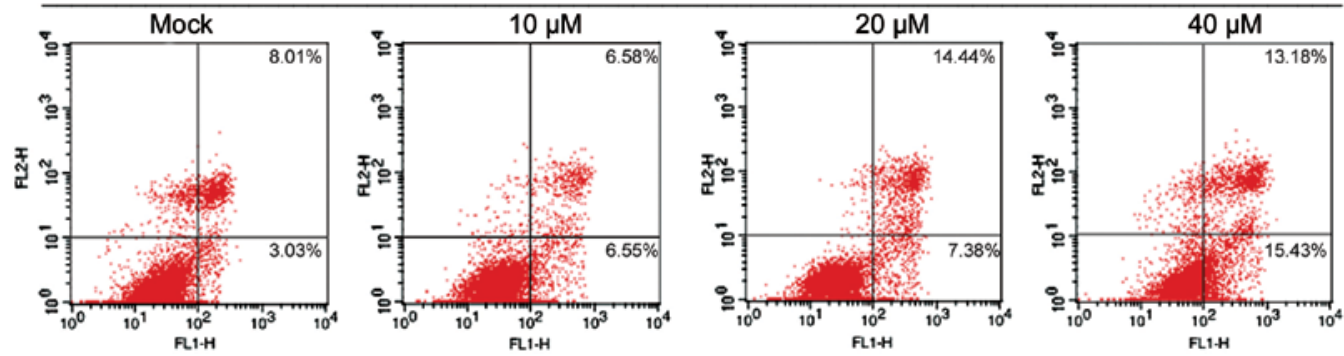

Figure 2. $2 \mathrm{HF}$ induces cell apoptosis in metastatic CRPC cells. (A) PC-3 cells were treated with different doses of $2 \mathrm{HF}(0,20$ and $40 \mu \mathrm{mol} / 1)$ for $24 \mathrm{~h}$ and then subjected to Annexin V/PI staining and flow cytometric analysis. Representative images of flow cytometry are shown. (B) DU145 cells were treated with different doses of $2 \mathrm{HF}(0,20$ and $40 \mu \mathrm{mol} / \mathrm{l})$ for $24 \mathrm{~h}$ and then subjected to Annexin V/PI staining and flow cytometric analysis. Representative images of flow cytometry are shown. (C) C4-2 cells were treated with different doses of $2 \mathrm{HF}(0,20$ and $40 \mu$ mol/1) for $24 \mathrm{~h}$ and then subjected to Annexin V/PI staining and flow cytometric analysis. Representative images of flow cytometer are shown.

performed a colony formation assay to analyze the potential effects of $2 \mathrm{HF}$ on the viability of PC-3 cells. Indeed, $2 \mathrm{HF}$ significantly inhibited the clonogenicity of PC-3 cells with a decreased number of colonies, in a dose-dependent manner (Fig. 1C). To rule out cell-specific effects, we repeated our MTT and colony formation assays in another PCa cell line, DU145, which was also androgen-independent and brain metastatic. Consistently, $2 \mathrm{HF}$ suppressed in vitro cell proliferation and clonogenicity of DU145 cells in a dose-dependent manner (Fig. 1B and C). Moreover, similar results were also observed in another bone metastatic, androgen-independent but androgen receptor (AR)-positive PCa cell line C4-2 (Fig. 1D), since the PC-3 and DU145 cells were AR-negative. All of these findings suggest that $2 \mathrm{HF}$ targets androgen-independent CRPC tumor growth in vitro, which does not depend on the status of $\mathrm{AR}$ expression.

$2 \mathrm{HF}$ induces cell apoptosis in prostate cancer cells. Apoptosis is one of the important mechanisms by which anticancer agents inhibit the growth of cancer cells. To understand the mechanism by which $2 \mathrm{HF}$ inhibits the viability of prostate cancer cells, PC-3 and DU145 cells were exposed to various concentrations of $2 \mathrm{HF}$ for $24 \mathrm{~h}$, and the population of apopototic cells after Annexin V/PI staining was determined by flow cytometry. As the representative data show in Fig. $2 \mathrm{~A}, 2 \mathrm{HF}$ treatments at 10, 20 and $40 \mu \mathrm{M}$ resulted in 8.82, 11.85 and $21.5 \%$ apoptotic cells in the PC-3 cells, respectively, while the baseline of the control cells was $7.35 \%$. In addition, $2 \mathrm{HF}$ treatment at 10, 20 and $40 \mu \mathrm{M}$ induced $6.42,12.75$ and $27.39 \%$ apoptosis in the DU145 cells, respectively, while the baseline of the control cells was $5.45 \%$ (Fig. 2B). Moreover, similar results were observed in C4-2 cells after 2HF treatment (Fig. 2C). Correspondingly, $2 \mathrm{HF}$ gradually increased the cleavage of poly-PARP, a chromatin-associated enzyme that plays an important role in DNA repair and the recovery of cells from DNA damage, in a dose-dependent manner in both PC-3 and DU145 cells (Fig. 3A and B). Consistent with the results, total caspase-3 proteins decreased accordingly after $2 \mathrm{HF}$ treatment, indicating the cleavage of caspase-3. Collectively, these results indicate that the induction of apoptotic cell death by $2 \mathrm{HF}$ probably occurs through a caspase-dependent pathway.

$2 H F$ suppresses AKT/STAT3 signaling and the expression of the BCL-2 family in prostate cancer cells. To further investigate the mechanisms of $2 \mathrm{HF}$ targeting prostate tumor growth, we performed western blot analyses to detect the expression of proteins related to apoptosis. Indeed, $2 \mathrm{HF}$ treatment modulated the expression of members of the $B C L-2$ 
A

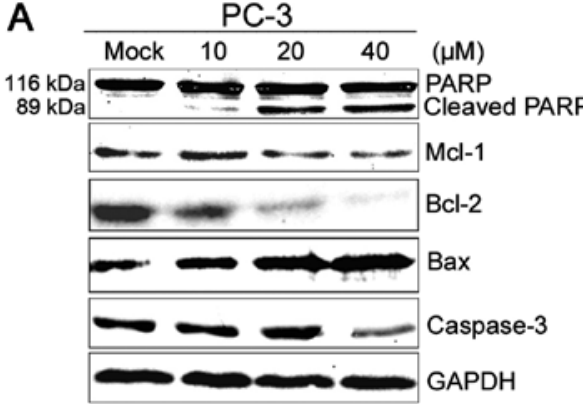

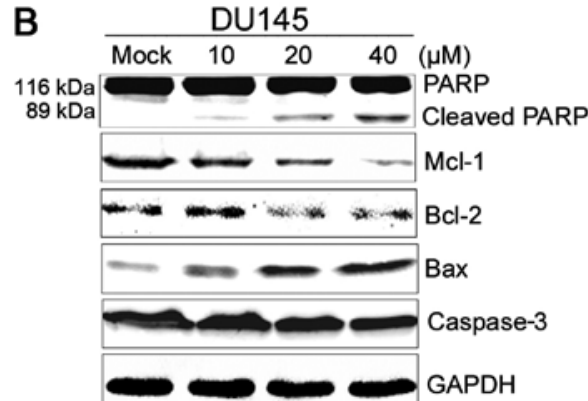

Figure 3. 2HF modulates the expression of BCL-2 family members and induces the cleavage of PARP and caspase-3. (A) PC-3 cells were treated with different doses of $2 \mathrm{HF}(0,20$ and $40 \mu \mathrm{mol} / \mathrm{l})$ for $36 \mathrm{~h}$ and then cell lysates were harvested for western blot analyses to detect the expression of cleaved PARP, caspase-3, Mcl-1, Bcl-2 and Bax proteins. GAPDH was used as a loading control. Representative images of the bands are shown. (B) DU145 cells were treated with different doses of $2 \mathrm{HF}(0,20$ and $40 \mu \mathrm{mol} / \mathrm{l})$ for $36 \mathrm{~h}$ and then cell lysates were harvested for western blot analyses to detect the expression of cleaved PARP, caspase-3, Mcl-1, Bcl-2 and Bax proteins. GAPDH was used as a loading control. Representative images of the bands are shown.

A

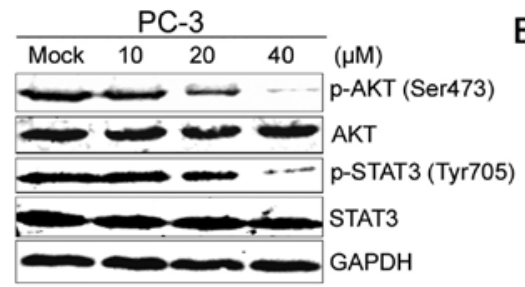

C

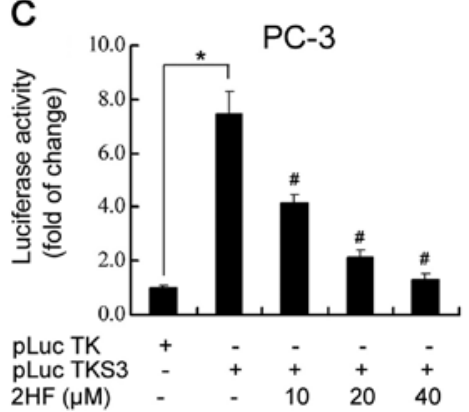

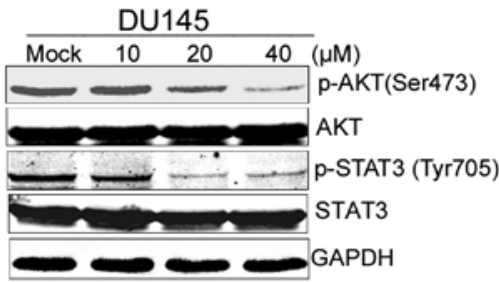

D

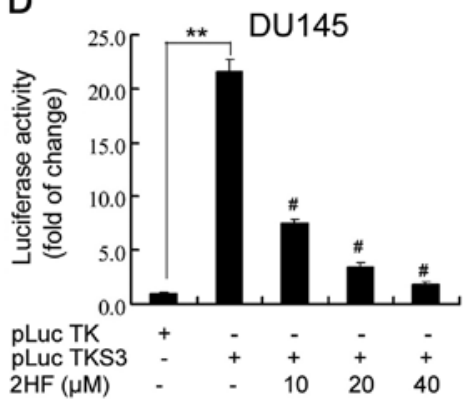

Figure 4. 2HF suppresses the phosphorylation of AKT and STAT3 and then inhibits STAT3 transcription activity. (A and B) PC-3 and DU145 cells were treated with different doses of $2 \mathrm{HF}(0,20$ and $40 \mu \mathrm{mol} / \mathrm{l})$ for $36 \mathrm{~h}$ and then cell lysates were harvested for western blot analyses to detect the expression of $\mathrm{p}$-AKT (Ser473), total AKT, p-STAT3 (Tyr705) and total STAT3 protein. GAPDH was used as a loading control. Representative images of the bands are shown. (C and D) PC-3 and DU145 cells were transfected with pLucTKS3 or nonresponsive control reporter pLucTK and internal control pRL-SV40 Renilla luciferase construct for $12 \mathrm{~h}$ and then treated with different doses of $2 \mathrm{HF}$ for $36 \mathrm{~h}$ before dual-luciferase assay. Relative luciferase activity is represented as the mean \pm SEM of each sample after normalizing with the control $(=1),{ }^{*} \mathrm{P}<0.05,{ }^{* *} \mathrm{P}<0.01$ between indicated groups, ${ }^{*} \mathrm{P}<0.05$ compared with the control.

gene family (15), which include protective proteins involved in the mitochondrial apoptotic pathway. As shown in Fig. 3A, following the treatment of PC-3 cells with 10,20 and $40 \mu \mathrm{M}$ $2 \mathrm{HF}$ for $48 \mathrm{~h}$, the expression levels of $\mathrm{Mcl}-1$ and $\mathrm{Bcl}-2$ proteins were significantly downregulated in a dose-dependent manner, while Bax protein was gradually upregulated after treatment. Similar results were also observed in the DU145 cells (Fig. 3B).

PTEN deletions and mutations lead to aberrant activation of the PI3K/AKT pathway in prostate cancer (16), which is involved in the regulation of cancer cell growth, motility, survival and metabolism. Thus inactivation or inhibition of PI3K/AKT signaling pathways provide a new substantial strategy to target CRPC using small molecules or plant extracts $(17,18)$. In the present study, we observed that $2 \mathrm{HF}$ treatment markedly inhibited the phosphorylation of AKT on the site of serine 473 in both PC-3 and DU145 cells (Fig. 4A and $\mathrm{B})$. $2 \mathrm{HF}$ treatment also inhibited the phosphorylation of
STAT3 (Fig. 4A and B), another critical signaling transduction protein, which is constitutively activated in prostate cancer by phosphorylation of its tyrosine 705 residue (19). After phosphorylation, STAT3 homodimerizes and translocates to the nucleus where it binds to specific STAT3 response elements of target gene promoters to regulate transcription. Indeed, using a specific STAT3-responsive luciferase reporter (13), we further demonstrated that $2 \mathrm{HF}$ suppressed STAT3 transactivation in both PC-3 and DU145 cells based on a luciferase reporter gene assay, which was also dose-dependent (Fig. 4C and D). Therefore, $2 \mathrm{HF}$ inhibited AKT/STAT3 signaling and the expression of the BCL-2 family in the prostate cancer cells.

AKT/STAT3 signaling regulates Bcl-2 expression and cell apoptosis after $2 \mathrm{HF}$ treatment. To further dissect the roles of AKT and STAT3 signaling in the inhibitory effects of $2 \mathrm{HF}$ on PCa cells, we applied the PI3K inhibitor LY294002 or 
A

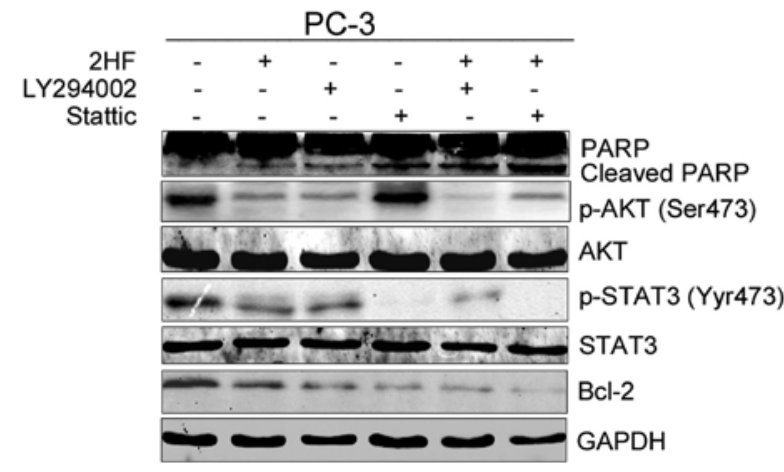

B

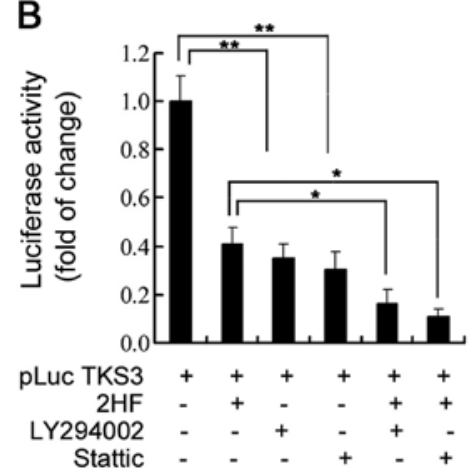

C

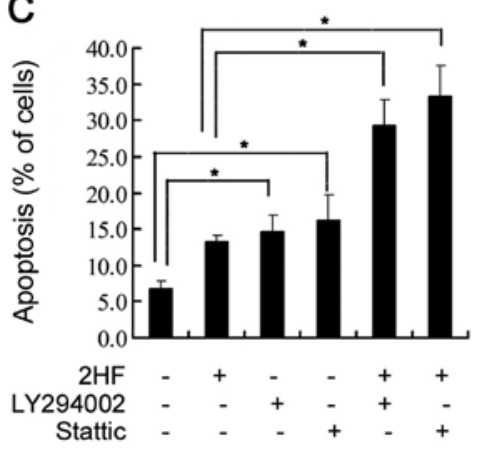

Figure 5. 2HF regulates Bcl-2 expression and cell apoptosis through AKT/STAT3 signaling. (A) PC-3 cells were pre-treated with 20 $\mu \mathrm{M}$ LY 294002, Stattic or DMSO for $30 \mathrm{~min}$ and then treated with $20 \mu \mathrm{mol} / 12 \mathrm{HF}$ for $36 \mathrm{~h}$ before cell lysates were harvested for western blot analyses to detect the expression of cleaved PARP, p-AKT (Ser473), total AKT, p-STAT3 (Tyr705) and total STAT3 protein. GAPDH was used as a loading control. Representative images of the bands are shown. (B) PC-3 cells were transfected with pLucTKS3 or nonresponsive control reporter pLucTK and internal control pRL-SV40 Renilla luciferase construct for $12 \mathrm{~h}$ and pre-treated with $20 \mu \mathrm{M} \mathrm{LY} 294002$, Stattic or DMSO for $30 \mathrm{~min}$ and then treated with $20 \mu \mathrm{mol} / 12 \mathrm{HF}$ for $36 \mathrm{~h}$ before dual-luciferase assay. Relative luciferase activity is represented as the mean \pm SEM of each sample after normalizing with the control $(=1),{ }^{*} \mathrm{P}<0.05,{ }^{* *} \mathrm{P}<0.01$ between indicated groups. (C) PC-3 cells were pre-treated with $20 \mu \mathrm{M} \mathrm{LY} 294002$, Stattic or DMSO for $30 \mathrm{~min}$ and then treated with $20 \mu \mathrm{mol} / 12 \mathrm{HF}$ for $36 \mathrm{~h}$ before Annexin V/PI staining and flow cytometric analysis. Quantitative analysis of the cell apoptotic rate from three independent experiments is shown. ${ }^{*} \mathrm{P}<0.05$ between indicated groups.

STAT3 inhibitor Stattic to pretreat PC-3 cells and analyzed the changes in Bcl-2 protein expression and cell apoptosis. As shown in Fig. 5A, western blotting data clearly indicated that LY294002 treatment alone significantly decreased p-AKT (Ser473), p-STAT3 (Tyr705) and Bcl-2 protein expression in the PC-3 cells and it also enhanced the inhibitory effects of $2 \mathrm{HF}$ on AKT phosphorylation, STAT3 phosphorylation and $\mathrm{Bcl}-2$ protein expression (Fig. 5A). Consistently, it also increased the inhibitory effects of 2HF on STAT3-responsive luciferase activity (Fig. 5B). Moreover, inhibition of PI3K/ AKT also significantly enhanced the induction of PC-3 cell apoptosis after 2HF treatment (Fig. 5C). In contrast, Stattic treatment had no effects on AKT phosphorylation in PC-3 cells (Fig. 5A), indicating that the STAT3 pathway was activated as downstream signaling of PI3K/AKT. However, inhibition of STAT3 activity also decreased Bcl-2 protein expression and increased cell apoptosis (Fig. 5A and C). Moreover, we observed that additional Stattic treatment further decreased Bcl-2 protein expression and increased cell apoptosis of PC-3 cells induced by $2 \mathrm{HF}$ treatment (Fig. 5A and C).

$2 \mathrm{HF}$ inhibits prostate tumor growth and AKT/STAT3/Bcl-2 signaling in vivo. We also generated xenograft models to show the inhibitory effects of $2 \mathrm{HF}$ on prostate tumor growth in vivo. As shown in Fig. 6A, oral $2 \mathrm{HF}$ treatment significantly decreased the tumor burden of subcutaneous PC-3 xenografts (left and middle panel), and the average tumor volume was much smaller (right panel, $\mathrm{P}<0.05$ ). Furthermore, IHC staining data also supported the observation in the cell lines in vitro. p-AKT (Ser473), p-STAT3 (Tyr705) and Bcl-2 protein expression levels in the subcutaneous PC-3 xenograft tissues with or without $2 \mathrm{HF}$ treatment were detected by IHC staining. Consistent with our findings in vitro, higher expression levels of p-AKT (Ser473), p-STAT3 (Tyr705) and Bcl-2 were detected in the PC-3 xenograft samples, indicating hyperactivation of AKT/STAT3/Bcl-2 signaling in metastatic CRPC, while $2 \mathrm{HF}$ treatment suppressed the phosphorylation of AKT and STAT3 and potentially inactivated STAT3 transcriptional activity and decreased the expression of its target gene Bcl-2 (Fig. 6B). We also observed that $2 \mathrm{HF}$ treatment increased the cleavage of caspase- 3 in the PC-3 xenografts using an IHC-specific cleaved-caspase-3 antibody (Fig. 6B), indicating the incidence of cell apoptosis in vivo after $2 \mathrm{HF}$ treatment.

\section{Discussion}

Treatment paradigms for men with metastatic CRPC have changed dramatically in the last three years due to the approval of agents such as the CYP-inhibitor abiraterone and secondgeneration AR antagonist enzalutamide, as well as other agents. These novel therapeutics have recently been proven to extend survival via distinct mechanisms of action (20). 

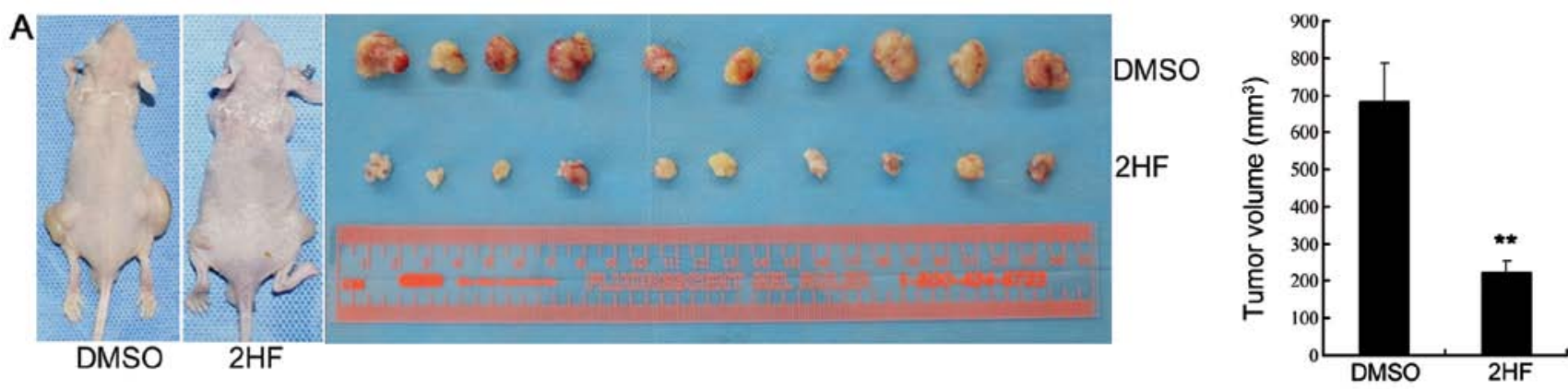

B

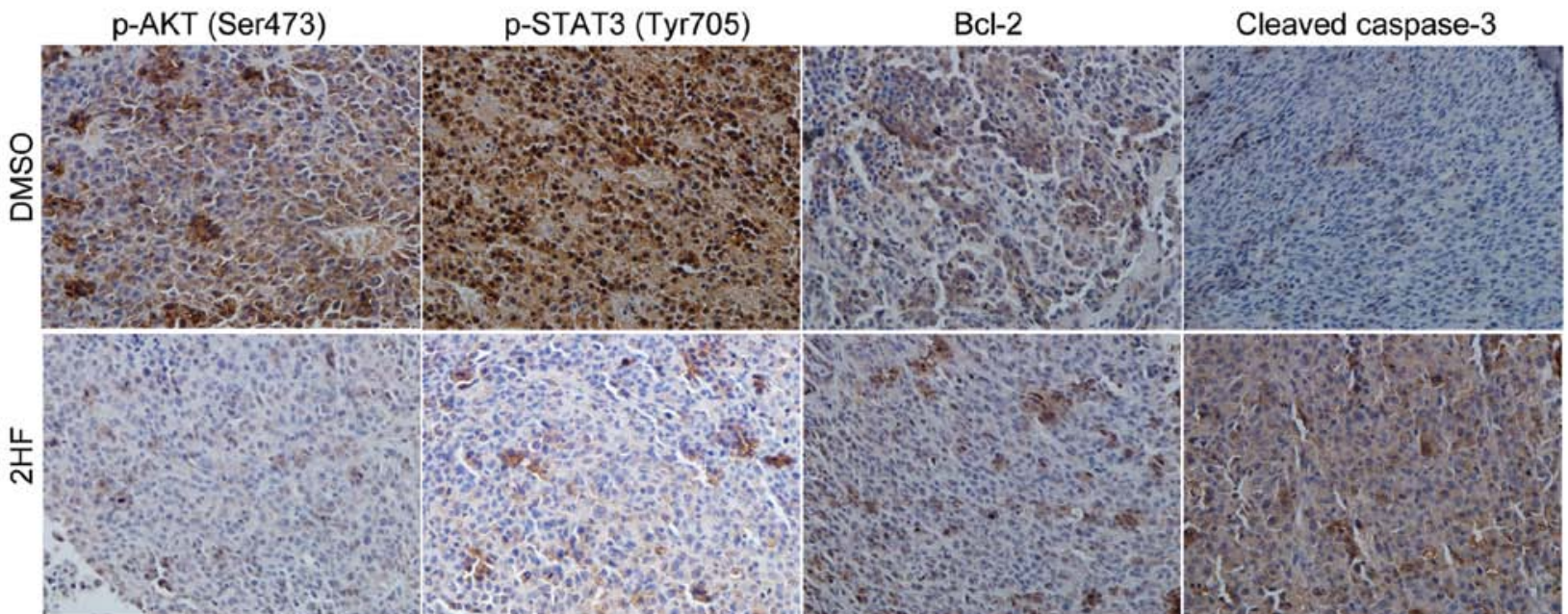

Figure 6. $2 \mathrm{HF}$ inhibits prostate tumor growth and AKT/STAT3/Bcl-2 signaling in vivo. (A) Left and middle panel, representative images of mice bearing PC-3 subcutaneous xenografts with or without $2 \mathrm{HF}$ treatment $(\mathrm{n}=10)$. Right panel, quantitative analysis of tumor weight; ${ }^{* *} \mathrm{P}<0.01$ between indicated groups (B) Representative images of the immunohistochemistry of p-AKR (Ser473), p-STAT3 (Tyr705), Bcl-2 and cleaved caspase-3 in PC-3 subcutaneous xenograft tissues after $2 \mathrm{HF}$ treatment.

However, drug resistance eventually develops; thus, further exploration into new adjuvant agents and their potential therapeutic mechanisms in prostate cancer are necessary.

Flavanones richly exist in citrus and have been well characterized to have various bioactive properties. In our previous reports (21-24), we extensively studied the chemopreventive and chemotherapeutic effects of silibinin, a flavanone isolated from the fruits of milk thistle, in prostate and bladder cancer cells. Here, we further observed that another nontoxic natural flavanone $2 \mathrm{HF}$ also significantly inhibited the cell proliferation and xenograft tumor growth of different metastatic CRPC cells (PC-3, DU145 and C4-2), in which the inhibitory effects of $2 \mathrm{HF}$ on cell growth and colony formation were dose- and time-dependent.

Previous research has shown that AR is highly expressed and transcriptionally active in CRPC and has indicated that steroids from the adrenal glands contribute to this AR activity (25). More recent studies revealed that CRPC cells have increased expression of enzymes mediating androgen synthesis from adrenal steroids and are also able to synthesize androgens de novo from cholesterol (26). Therefore, investigation of novel agents targeting these critical enzymes involved in de novo androgen synthesis is promising. A previous study in particular screened $2 \mathrm{HF}$ from several dietary flavonoids as a powerful selective inhibitor of AKR1C3, a critical aldo-keto reductase converting the weak androgen $\Delta 4$-androstene-3,17dione into the potent androgen testosterone, and as a novel potential target for CRPC treatment (27). Indeed, Ofude et al also further demonstrated that $2 \mathrm{HF}$ inhibited $\mathrm{AR}$ transcription activity, PSA expression and androgen-induced cell proliferation in LNCaP cells (28). However, most of our CRPC cell models (PC-3 and DU145) had no AR expression, suggesting that other androgen-AR bypass signaling may be involved in the inhibitory effects of $2 \mathrm{HF}$ on CRPC tumor growth.

CRPC cells maintain resistance to castration and recur after chemotherapy, which depends on the ratio of proliferating cells versus apoptotic cells. Therefore, the alteration of cell death signaling may still play an important role in novel PCa therapy (29). Indeed, our present study demonstrated that $2 \mathrm{HF}$ treatment significantly increased the cell apoptotic rate and cleavage of PARP and caspase-3 in PC-3 and DU145 cells in a dose-dependent manner, indicating that $2 \mathrm{HF}$ induces the incidence of caspase-mediated cell apoptosis in CRPC cells. A similar phenomenon has also been observed in other cancer cells, such as renal and colon carcinoma cells $(10,11)$. Moreover, a study by Ofude et al also demonstrated the induction of cell apoptosis in PC-3 and DU145 cells after 2HF treatment (28), yet the mechanisms remain largely unknown.

As known, the PI3K pathway is aberrantly activated in prostate cancers due to PTEN deletions and mutations and may also be involved in CRPC progression, which leads to the recruitment and activation of the AKT serine/threonine kinase (34) and subsequently regulates tumor cell proliferation, growth, survival and metastasis. Therefore, inactivation or inhibition of the PI3K/AKT signaling pathways provide a new substantial strategy for CRPC treatment. In the present study, 
we further demonstrated that $2 \mathrm{HF}$ significantly suppressed the phosphorylation of AKT on the site of serine473. Furthermore, inactivation of AKT after 2HF treatment in turn inhibited the phosphorylation and transactivation of STAT3, indicating that 2HF targets distinct signaling cascades for CRPC treatment.

As a constitutively active transcription factor in CRPC, STAT3 becomes active by phosphorylation of a specific tyrosine residue in the carboxy-terminal domain by a tyrosine kinase (Tyr705) and then homodimerizes and translocates to the nucleus where it binds to specific STAT3 response elements of target gene promoters to regulate transcription (13). Indeed, our results also revealed that $2 \mathrm{HF}$ modulated the expression of several apoptotic and anti-apoptotic genes, Mcl-1, Bcl-2 and Bax, which have been reported as typical downstream target genes of STAT3 (30). Therefore, consistent with our previous study reporting the cross-talk of PI3K/AKT and STAT3 signaling regulating $\mathrm{Bcl}-2$ expression in CRPC development (31), 2HF suppressed the activation of AKT and STAT3 and then modulated the expression of members of the $B C L-2$ gene family in vitro and in vivo, which are critical for regulating mitochondrial apoptosis.

Taken together, our research revealed a novel mechanism for $2 \mathrm{HF}$ targeting metastatic CRPC tumor growth in vitro and in vivo, in which inactivation of AKT/STAT3 by $2 \mathrm{HF}$ treatment led to a change in expression of the BCL-2 gene family members and then induced cell apoptosis and growth inhibition. Therefore, our findings demonstrated that $2 \mathrm{HF}$ may be a novel potential agent for the prevention and therapy of metastatic CRPC, not only working as a selective inhibitor of AKR1C3 but also as a strong inducer of cell apoptosis.

\section{Acknowledgements}

This study was supported by the National Natural Science Foundation of China (NSFC 81202014 to K.W.) and the Fundamental Research Funds for the Central Universities (to K.W.).

\section{References}

1. Siegel R, Naishadham D and Jemal A: Cancer statistics, 2013 CA Cancer J Clin 63: 11-30, 2013.

2. Heidenreich A and Pfister D: Treatment decisions for metastatic castration-resistant prostate cancer progressing after docetaxel chemotherapy: the role of cabazitaxel in the continuum of care. Eur Urol 62: 1201-1204, 2012.

3. Beltran H, Beer TM, Carducci MA, et al: New therapies for castration-resistant prostate cancer: efficacy and safety. Eur Urol 60: 279-290, 2011.

4. Mottet N, Bellmunt J, Bolla M, et al: EAU guidelines on prostate cancer. Part II: Treatment of advanced, relapsing, and castrationresistant prostate cancer. Eur Urol 59: 572-583, 2011.

5. Vitale DC, Piazza C, Melilli B, Drago F and Salomone S: Isoflavones: estrogenic activity, biological effect and bioavailability. Eur J Drug Metab Pharmacokinet 38: 15-25, 2013.

6. Ko CH, Shen SC, Lin HY, et al: Flavanones structure-related inhibition on TPA-induced tumor promotion through suppression of extracellular signal-regulated protein kinases: involvement of prostaglandin E2 in anti-promotive process. J Cell Physiol 193: 93-102, 2002.

7. Romagnolo DF and Selmin OI: Flavonoids and cancer prevention: a review of the evidence. J Nutr Gerontol Geriatr 31: 206-238, 2012.

8. Manthey JA, Grohmann K and Guthrie N: Biological properties of citrus flavonoids pertaining to cancer and inflammation. Curr Med Chem 8: 135-153, 2001.
9. Hsiao YC, Kuo WH, Chen PN, et al: Flavanone and 2'-OH flavanone inhibit metastasis of lung cancer cells via down-regulation of proteinases activities and MAPK pathway. Chem Biol Interact 167: 193-206, 2007.

10. Nagaprashantha LD, Vatsyayan R, Singhal J, et al: 2'-hydroxyflavanone inhibits proliferation, tumor vascularization and promotes normal differentiation in VHL-mutant renal cell carcinoma. Carcinogenesis 32: 568-575, 2011.

11. Shin SY,KimJH,Lee JH,Lim Y andLee YH: 2'-Hydroxyflavanone induces apoptosis through Egr-1 involving expression of Bax, p21, and NAG-1 in colon cancer cells. Mol Nutr Food Res 56: 761-774, 2012.

12. Wu K, Gore C, Yang L, et al: Slug, a unique androgen-regulated transcription factor, coordinates androgen receptor to facilitate castration resistance in prostate cancer. Mol Endocrinol 26: 1496-1507, 2012.

13. Turkson J, Bowman T, Garcia R, Caldenhoven E, De Groot RP and Jove R: Stat 3 activation by Src induces specific gene regulation and is required for cell transformation. Mol Cell Biol 18: 2545-2552, 1998 .

14. Wu K, Fan J, Zhang L, et al: PI3K/Akt to GSK3 $\beta / \beta$-catenin signaling cascade coordinates cell colonization for bladder cancer bone metastasis through regulating ZEB1 transcription. Cell Signal 24: 2273-2282, 2012.

15. Youle RJ and Strasser A: The BCL-2 protein family: opposing activities that mediate cell death. Nat Rev Mol Cell Biol 9: 47-59, 2008.

16. Majumder PK and Sellers WR: Akt-regulated pathways in prostate cancer. Oncogene 24: 7465-7474, 2005.

17. Nelson EC, Evans CP, Mack PC, Devere-White RW and Lara PN Jr: Inhibition of Akt pathways in the treatment of prostate cancer. Prostate Cancer Prostatic Dis 10: 331-339, 2007.

18. Morgan TM, Koreckij TD and Corey E: Targeted therapy for advanced prostate cancer: inhibition of the PI3K/Akt/mTOR pathway. Curr Cancer Drug Targets 9: 237-249, 2009.

19. Mora LB, Buettner R, Seigne J, et al: Constitutive activation of Stat 3 in human prostate tumors and cell lines: direct inhibition of Stat 3 signaling induces apoptosis of prostate cancer cells. Cancer Res 62: 6659-6666, 2002.

20. Gaya JM, Ahallal Y, Sanchez-Salas R, et al: Current, new and novel therapy for castration-resistant prostate cancer. Expert Rev Anticancer Ther 13: 819-827, 2013.

21. Wu KJ, Zeng J, Zhu GD, et al: Silibinin inhibits prostate cancer invasion, motility and migration by suppressing vimentin and MMP-2 expression. Acta Pharmacol Sin 30: 1162-1168, 2009.

22. Wu K, Zeng J, Li L, et al: Silibinin reverses epithelial-to-mesenchymal transition in metastatic prostate cancer cells by targeting transcription factors. Oncol Rep 23: 1545-1552, 2010.

23. Zeng J, Sun Y, Wu K, et al: Chemopreventive and chemotherapeutic effects of intravesical silibinin against bladder cancer by acting on mitochondria. Mol Cancer Ther 10: 104-116, 2011.

24. Wu K, Ning Z, Zeng J, et al: Silibinin inhibits $\beta$-catenin/ZEB1 signaling and suppresses bladder cancer metastasis via dualblocking epithelial-mesenchymal transition and stemness. Cell Signal 25: 2625-2633, 2013.

25. Stanbrough M, Bubley GJ, Ross K, et al: Increased expression of genes converting adrenal androgens to testosterone in androgenindependent prostate cancer. Cancer Res 66: 2815-2825, 2006.

26. Yuan X, Cai C, Chen S, Yu Z and Balk SP: Androgen receptor functions in castration-resistant prostate cancer and mechanisms of resistance to new agents targeting the androgen axis. Oncogene: Jun 10, 2013 (Epub ahead of print). doi: 10.1038/onc.2013.235.

27. Adeniji AO, Chen M and Penning TM: AKR1C3 as a target in castrate resistant prostate cancer. J Steroid Biochem Mol Biol 37: 136-149, 2013.

28. Ofude M, Mizokami A, Kumaki M, et al: Repression of cell proliferation and androgen receptor activity in prostate cancer cells by 2'-hydroxyflavanone. Anticancer Res 33: 4453-4461, 2013.

29. Zielinski RR, Eigl BJ and Chi KN: Targeting the apoptosis pathway in prostate cancer. Cancer J 19: 79-89, 2013.

30. Real PJ, Sierra A, De Juan A, Segovia JC, Lopez-Vega JM and Fernandez-Luna JL: Resistance to chemotherapy via Stat3-dependent overexpression of Bcl-2 in metastatic breast cancer cells. Oncogene 21: 7611-7618, 2002.

31. Zhang D, He D, Xue Y, et al: PrLZ protects prostate cancer cells from apoptosis induced by androgen deprivation via the activation of Stat3/Bcl-2 pathway. Cancer Res 71: 2193-2202, 2011. 